\title{
The promise of dasymetric maps for monitoring progress towards the United Nations Sustainable Development Goals
}

\author{
Britta Ricker $^{\mathrm{a}, *}$, Menno Jan Kraak ${ }^{\mathrm{b}}$, Robert E. Roth ${ }^{\mathrm{c}}$ \\ ${ }^{a}$ Utrecht University, b.a.ricker@uu.nl, \\ ${ }^{b}$ University of Twente, m.j.kraak@utwente.nl \\ ${ }^{c}$ University of Wisconsin, reroth@wisc.edu \\ * Corresponding author
}

Keywords: Thematic maps, Dasymetric, Sustainable Development Goals

\begin{abstract}
:
To achieve the 17 ambitious Sustainable Development Goals (SDG) set by the United Nations (UN), 169 targets and over 200 indicator datasets have been identified to monitor progress to achieve these goals worldwide. The aim of these goals is to find actionable localized solutions to wicked problems such as poverty, hunger, access to water, environmental justice, gender equality, and more. Maps are essential for identifying where to focus efforts to achieve these goals (Kraak, et al., 2018). However, all SDG indicator datasets are aggregated at the country level, meaning thematic map types that are most appropriate for mapping these data are limited. For data that can be normalized, choropleth maps are a common choice. However, choropleth maps lead the map reader to believe that a phenomenon is evenly distributed across an administration unit, in the case of the SDGs, a country. This makes it impossible to detect precisely where tailored localized solutions could be administered. Therefore, alternative thematic map types could better represent the SDG indicators to find where people are being affected and where interventions need to take place (Gosling et al., 2020, Pirani et al, 2020). One map type that could be particularly useful at portraying a more accurate representation of a phenomena are dasymetric maps.
\end{abstract}

Like choropleth maps, dasymetric maps can use area symbols to represent zones of uniformity, but instead redraw the often arbitrary political boundaries used for SDG mapping into borders more meaningful to mapped phenomenon (Kraak et al, 2020). The United Nations does not provide ancillary information to make dasymetric maps, and the best quality ancillary data likely is specific to each nation's spatial data infrastructure, making dasymetric mapping at a global scale difficult if not impossible. Attempting dasymetric maps is still worthwhile as dasymetric mapping could provide a more realistic form of data presentation for many SDG indicators since it displays a more realistic version of the geographic distribution of where specific phenomena occur, such as where a forest is located or where specific populations live that do not have access to sanitation services. With this approach to dasymetric mapping, the map maker uses land cover datasets as ancillary data to reassign the original data to different enumeration units within the map. As a result, the normalized values change since the data are redistributed across different areas in the dasymetric map compared to the choropleth format. Since global SDG indicators provide only a coarse understanding of disparities in environmental sustainability, social inclusion, and economic prosperity, utilizing ancillary data are required to make dasymetric maps. As a result, these maps can better support localized monitoring and policymaking in lieu of higherresolution subnational datasets harmonized across countries.

Dasymetric maps redraw boundaries using exclusionary or inclusionary ancillary datasets. Exclusionary data defines locations where the mapped phenomenon cannot exist. For instance, trees cannot grow above a certain elevation, in a desert receiving a minimal amount of precipitation, or in a lake or river. Thus elevation, annual precipitation, and land cover can be used as exclusionary factors in a dasymetric map of protected forests. Inclusionary data defines locations where the mapped phenomenon can exist, often in different amounts. For example, rural versus urban areas can be used to measure the existence of a given social phenomenon such as poverty based on the average population densities for specific census or other demarcation zones in a given country (e.g., 20 people per $\mathrm{km}$ in rural, 2,000 people per km in urban). We present the way in which we generated a dasymetric map for our book Mapping for a Sustainable World (see Figure 4 below and Kraak et al, 2020: 67). Here, we selected the indicator 2.1.1 "Prevalence of undernourishment", undernourished people per km2, in four countries in Sub-Saharan Africa. We selected this region because it is suffering significantly from violence, draughts, poverty and undernourishment and wanted to better understand where undernourishment may be occurring. We wanted to represent the spatial distribution of the rates of undernourishment 
based on where the majority of people are living in this region. Our aim was to offer a more precise understanding of the spatial unevenness of food security in the region.

Arguably, it is possible to make dasymetric maps manually or digitally using Geographic information Systems (GIS) to recalculate areas. To generate this dasymetric map, first we used GIS to calculate the absolute values for each or the original enumeration unit from the UN website, in the case of the SDGs by country and then normalized relative to the area. In other words, we created a traditional choropleth map to begin the dasymetric process (Figure 1). Next, we identified relevant ancillary data to locate areas where the mapped phenomenon likely does and does not exist. We identified a land use classification additional data; the most readily available Global Land Cover dataset from the European Commission for the year 2000 was used (Figure 2). Then, the original enumeration units were dissolved into subareas for exclusion of the mapped phenomenon - in this case, we used raster-based land use data and excluded dessert and water bodies (Figure 3). We then redistributed the absolute values from the indicator data to these finer resolution boundaries, exclusionary areas getting no value and inclusionary areas given values based on predetermined weights which are the raw number normalized by the new calculated area. Finally, we normalized the data by redistributed absolute values into the new calculated areas (minus desert and water areas), producing a new set of shaded areas that provide greater detail about the spatial distribution of the SDG indicator (Figure 4). We now invite you to get creative and make your own dasymetric maps. We argue for more imaginative solutions to determine better ways to portray the SDG indicator data in an effort to meet the goals themselves. We hope that in the future more localized data collection and data sharing are possible to make more appropriate maps to help meet the valuable SDGs, in a global effort to leave no one behind.
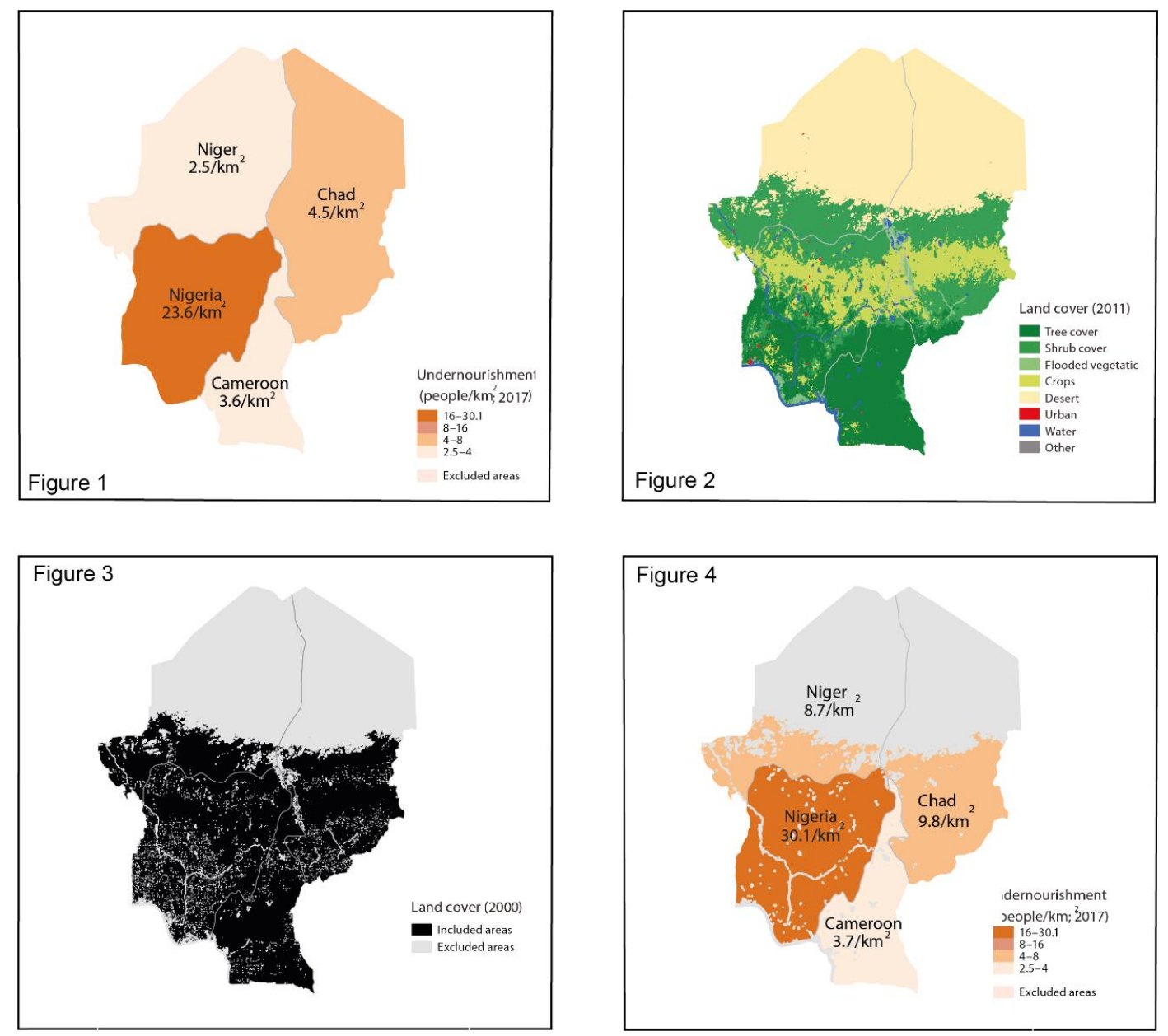

Figure 1: Original choropleth map. Indicator 2.1.1 (2017) the number of undernourished people per km2 is displayed for four countries in Sub-Saharan Africa. Figure 2: Exclusionary data. Classified land cover (2000) processed from satellite imagery is used to identify areas where people do not live: desert and water. Figure 3: Generating the clipping mask. Vector math was applied to remove the desert and water classes from the enumeration unit. Large areas in the north are removed due to the location of the Sahara Desert in addition to smaller water bodies such as Lake Chad and the Niger River. Figure 4: Resulting dasymetric map. Indicator 2.1.1 (2017) the number of undernourished people per $\mathrm{km} 2$ was remapped with the recalculated area as the denominator. As a result, Niger and Chad have a much greater density, pushing them into higher choropleth classes. A version of this figure can be found in Kraak et al 2020 page 67. 


\section{References}

Global Land Cover 2000 database. European Commission, Joint Research Centre, 2003.

Gosling-Goldsmith, J., Ricker, B., \& Jan Kraak, M. 2020. Topographic and thematic (in)visibility of Small Island Developing States in a world map. Journal of Maps, 16(1), 50-56. https://doi.org/10.1080/17445647.2020.1736194.

Kraak, M. J., Ricker, B., \& Engelhardt, Y. 2018. Challenges of Mapping SDG Indicators Data. ISPRS International Journal of Geo-Information, 7(12), 482. https://doi.org/10.3390/ijgi7120482

Kraak MJ, RE Roth, B Ricker, A Kagawa, and G Le Sourd. 2020. Mapping for a Sustainable World. The United Nations: New York, NY (USA). https://digitallibrary.un.org/record/3898826? ln=en

Pirani, N., Ricker, B. A., \& Kraak, M. J. 2020. Feminist cartography and the United Nations Sustainable Development Goal on gender equality: Emotional responses to three thematic maps. Canadian Geographer, 64(2), 184-198. https://doi.org/10.1111/cag.12575. 